\title{
Teaching of lens design
}

\author{
Robert Shannon
}

Robert R. Shannon, "Teaching of lens design," Proc. SPIE 1603, Education in Optics, (1 March 1992); doi: 10.1117/12.57848

SPIE. Event: Education in Optics, 1991, Leningrad, Russian Federation 
Invited Paper

The Teaching of Lens Design

\author{
R. R. Shannon \\ Optical Sciences Center \\ University of Arizona
}

\title{
Introduction: What is Lens Design
}

Lens design is both the art and the science involved in the application of basic optical principles to the creation of a set of parameters describing a lens for an optical instrument employing ref racting or reflecting components. The techniques involved in lens design incorporate the setup of a system using basic optical principles, the iteration of that initial description using geometrical optical principles and the analysis of the system in terms of its ability to convey information or energy to a detector.

This general description of the subject indicates that lens design is a practical application of optical principles to the creation of an lens system that will meet a specified need. The teaching of the subject therefore requires the acquisition of specific subject related knowledge as well as instruction in the practical application of this knowledge.

Traditionally lens design required the immersion of the designer in a procedure that involved the use of a description of the aberrations of a lens verified by a minimum of detailed numerical ray tracing. The result was

Teaching of Lens design Goals

- Application of optical principles to an Engineering Problem

- Basic concepts Interpreted to meet the needs of the designer

- Practical experience and contact with real problems is needed

heavily dependent upon the experience and intuition of the designer. Today high speed computers have replaced the drudgery of hand computation with the opportunity to explore in great detail the many possible solutions for a design problem. The basic principles still apply, but the capability to rapidly explore many regions of solution space reduces the strong dependence upon the intuition of the designer.

Teaching of the subject must start with a development of the basic optical principles. In some respects this is a review of the simple optics learned earlier by a student, such as paraxial optics and the principles of interference and diffraction. However the "review" of these subjects must be directed toward the development of an integrated understanding of these concepts, as they will eventually be used as the foundation for the layout and design of lens systems.

The theoretical heart of optical design is the use of ray tracing to explore the solution space. Ray tracing couples system parameters, such as curvatures, separations and glass choices, to the aberrations or defects that result from a given configuration. In other words, the very complex nature of the imagery that is produced by a lens is

Basic Requirements Theoretical Base
- Ray Tracing
- Paraxial Optics
- Aberration Definition
- Wavefront Descriptions
- Diffraction Calculations

- Merit Function Construction determined by tracing large sets of rays and interpreting the numerical results of the ray tracing in terms of the image quality produced. 
The introduction of physical optics into design is accomplished by using a diffraction integral to compute the image intensity distribution from the wavefront errors computed by ray tracing. The most common model used is based upon the Fourier Transform of the pupil function. Correct computation requires that the pupil and image plane coordinates be properly specified.

There are some necessary theoretical foundations required by a skilled lens designer. Some of my favorite topics include vector ray tracing, spherical pupil concepts relating paraxial and real ray optics, linearity definition of basic

Special Theoretical Basis Required for Designers

- Spherical Pupil Geometry

- Linearity Summation in Paraxial Traces

- Aberration Polynomial definition

- Extended Lagrange Concept

- Small aberration effect on MTF

- Tolerancing techniques paraxial concepts, and aberration polynomial descriptions of wavef ront errors. In addition the topics of power component distribution of aberrations, merit function definition using minimum ray sets and the definition of isoplanatic systems using the extended Lagrange invariant concept are important. For practical evaluation, the small aberration description of MTF changes for use in tolerancing and system performance prediction are important.

Approach to teaching

Lens design can be taught at different levels. At the Optical Sciences Center, graduate students are required to take a number of core courses, a few of which cover the fundamentals of paraxial optics and provide some introduction to aberration theory. In general, these courses permit the graduate to be capable of specifying optical systems, and evaluating the result of a lens designer's work. One specific course is devoted to a study of the practice of lens design. In that course the use of computer programs to carry out the optimization of optical systems is stressed, along with an integrated presentation of the theory behind the decisions necessary in lens design.

Approach to Teaching
- Basic Core Courses
- Specific Lens Design Course
- Problem Oriented

The computer optimization methods that have been developed have reached a high degree of sophistication. Therefore, the choice has been to provide students with access to resources that are likely to be available to them after they complete their course and enter industry. Actual hands-on experience with real problems is felt to be of great importance.

\section{The Curriculum}

The organization of a course on lens design is based upon actual design problems. The problems are selected to provide an introduction to each of the basic principles in design with increasing complexity introduced within each subsequent problem. Each problem should also incorporate the lessons learned in the preceding problems. This provides a realistic simulation of the environment within which the lens designer operates.

The course begins with the student writing a basic meridional ray trace and applying it to a specified simple lens. The point of this exercise is that the student will become aware of the methods used in ray tracing on a computer, and develop an appreciation of the data obtained from such a computation. The sophistication of commercially available programs suggests that little time be spent here, but the exercise serves as an introduction into computer use. 
Following this ray trace introduction, the students are subjected to several problems covering the layout of optical systems applying first order principles. These are of increasing complexity, leading to one which has no single well defined solution. This lens layout, which requires the layout of a variable magnification, or zoom, system, introduces the students to the concept that not all design problems are soluble to the extent specified by the customer. This is usually the first time in their careers that students become aware that the "best" or "optimum" solution is not necessarily well defined, and that some regions of the design space will not be accessible because of boundary conditions.

Completion of this sequence of problems leads to an exhaustive study of the axial and narrow field design and performance evaluation of a doublet, both cemented and broken contact. The stress here is on the development from first order thin lens solution to a corrected lens with manufacturable properties. Especially important is the understanding of aberration balancing in the presence of chromatic variation of aberrations. This is explored fully in these designs without the necessity of learning how to compromise the aberration balance to obtain an optimum balance across a wide field. The initial lens is required to be rebalanced for different spectral regions. This portion of the problems breaks with the simplified traditional theory about color correction that the students have been taught in earlier courses and teaches some flexibility in thinking about glass selection and lens layout.

A three glass apochromat is then worked on. A first order solution can easily be found. However, the intent of this problem is to produce a lens that actually provides better image quality than the doublet. The intrinsic variation of spherical aberration with wavelength is dominant in this design, requiring more sophistication in the design approach than the doublet required.

Needless to say, the lectures that accompany the problems need to be reasonably correlated with the problems in order that the students have at hand the knowledge necessary for the current problem set. This is not always possible, as I have found that the acceptance rate of knowledge by each group of students is variable. The goal is to provide knowledge and understanding and have the student learn during problem solving how to apply this knowledge.

By this time the students should have learned how to define a merit function, how to follow an iterative process, and how to evaluate the image produced by their designs. Most important, they will have learned how to determine when the design has reached completion, and also when further improvement in the image quality is likely to require a significant amount of design time.

All of this is on the axial image region, so far. The next step is to expand the experience to a large field of view.

Several new concepts have to be introduced. The theory in the lectures must cover the origin of off axis aberrations on individual surfaces, and develop the relation between image field curvature, or Petzval sum, and the power distribution required in a lens. The balancing of field aberrations is, of course, essential to the understanding of the working of a wide field optical system.

Theoretical concepts regarding off axis aberrations are best explained using the auxiliary axis concept. 
A tremendous amount of understanding can be obtained from using the aberrations introduced at a single surface as a graphical base for understanding the aberration coupling concept.

The problems used to introduce these concepts start with the investigation of the field characteristics of a meniscus singlet with stop moved away from the lens. This is the well known "landscape lens" problem. The students are expected to use the computer to verify the theory, and to explore how to find solutions for a flattened tangential field. The difference between thin and thick lenses is also examined as a part of this problem. The relation between spherical aberration and astigmatism contribution in such a lens serves as an introduction to the next set of problems in which a photographic type of lens is designed.

The next problem is the major one for the term and is the thorough investigation of the design of a Triplet type objective. This is initially approached as a layout exercise to obtain a controlled Petzval sum, and paraxial color correction. After an initial evaluation and aberration

\begin{tabular}{|l|}
\hline The Curriculum \\
Lens Design, Continued \\
- Stop Shifted Meniscus \\
Symmetric lens \\
- Triplet lens \\
Setup \\
Third order \\
High order balance \\
Image evaluate \\
- Gauss Type Photo Objective \\
- Tolerancing \\
- Reflective and Aspheric Systems
\end{tabular}
balancing on a computer program, realistic thicknesses are introduced, and serious design proceeds. In this problem, glass choice, variation of the starting point and construction of a successful wide field merit function is learned. Comparison of the effectiveness of a used defined merit function to the default merit functions of some of the available optical design programs is carried out.

The final balancing of aberrations to obtain an optimum solution, and evaluation of the image qulity in the optimum solution against a set of overall system specifications is then completed. In general, the choice of the system specifications is made such that the accomplishment of the design to image quality specifications is barely possible. The students are told to find the "best possible solution" within the time limits allowed for working on the problem. The lessons learned during this portion of the work are related to the ability to select efficient methods of defining aberrations, relating these to the needs of the problem, and exercising judgement as to when to halt the design process.

As a comparison, the students then evaluate the design of a Gauss type photographic objective which is supplied to them. The redesign to meet specific field and aperture requirements is carried out. This problem provides a very realistic simulation of a commercial design problem, and provides some experience in meeting complex image quality specifications.

The next and follow-up problem covers the tolerancing of either the Gauss type or the triplet design. The student is requested to set a set of reasonable goals for image quality, and to evaluate the tolerance distribution on the lens parameters that allows meeting these specifications. The student also is required to relate these to reasonable shop specifications.

Finally, the important topic of reflective systems and aspheric surfaces is introduced. The evaluation of a paraboloid, the design and evaluation of an equivalent Classical Cassegrain and field widened Cassegrain and the layout of a Schmidt system are the last problems in the course.

As is obvious, there is a lot of work for the student involved in this one term course. The students spend many hours at the computer, and, hopefully, at least as many thinking about the problems they are working on. The students who complete the course are prepared to enter into a career in optical design. The problems have been selected and used to provide somewhat of a simulation of the real 
world of lens design, while being directed toward teaching the fundamental optics involved in the imaging process.

\section{Subsequent Courses}

The student finishing the above course is reasonably well equipped to enter into lens design as a profession. There are those who wish to specialize in the field, and to investigate the problems associated with design in more detail. Advanced courses that are offered should be complementary, and build upon the foundation described above.

An advanced course is offered on a optional basis. The topics studied cover the fundamentals of optimization techniques used in modern computer programs, in depth theory of the origin and calculation of aberrations, and the

\begin{tabular}{|l|}
\hline \multicolumn{1}{c|}{$\begin{array}{c}\text { Subsequent Course } \\
\text { Advanced Lens Design }\end{array}$} \\
- Seminar style, heavy computation labs. \\
- Origin of aberrations \\
- Visual systems \\
- Wide angle systems \\
- Holographic optics \\
- Gradient index
\end{tabular}
basics of novel types of systems. In addition actual detailed problems involving wide angle objectives, visual systems, holographic optics, gradient index lenses, null lenses for optical testing and, occasionally, zoom lenses, are covered.

Such an advanced course is by nature less formal and is best taught in a seminar format, with heavy student participation. The computational work is also more intensive, and the computing facilities are of ten strained.

\section{Some biases}

Obviously the approach to the teaching of lens design that I have adopted is subject to a reasonable set of personal biases. These necessarily come through in the courses. Some, but probably not all, of these are the following:

Lens design is a practical engineering activity which must be related to real world problems.

Excellence in lens design is acquired through understanding of the fundamental principles of geometrical optics and wise use of the powerful computational tools that are now available.

The design of a lens is not a "hit or miss' activity, but needs to be guided to a successful conclusion

Paraxial optics as normally taught in textbooks is not adequate for detailed optical design. A full understanding of the relation between real rays and the paraxial basis for imagery is needed.

A thorough understanding of diffraction, image formation and image quality analysis is essential to evaluating the ending point for an optical design.

The need by industry for optical designers today is for those who have the breadth of knowledge to be adaptable to the design of novel systems.

A sensitivity to tolerances and fabrication processes is essential for a successful optical designer. 
There are more, but time does not permit addressing them here.

\section{Conclusions}

Optical design is a subject worthy of teaching in the proper manner. It is not a simple theoretical subject, nor is it a subject that can be left to the computer as a theoretical optimization procedure. Most of the old basic optical concepts are applicable today, but have to be tempered with the ability to fully and economically analyze optical designs on a computer. It is through an understanding of the mixture of these concepts, balanced with a proper application of computer techniques that a student can be turned into a successful lens designer. 